\title{
Role of Gamma Amino Butyric Acid (GABA) against abiotic stress tolerance in legumes: a review
}

\author{
Kumari Sita $^{1} \cdot$ Vaneet Kumar $^{2}$
}

Received: 19 September 2020/ Accepted: 24 November 2020/Published online: 9 December 2020

(C) Indian Society for Plant Physiology 2020

\begin{abstract}
Legumes are well known for their nutritional and health benefits as well as for their impact in the sustainability of agricultural systems. Under current scenarios threatened by climate change highlights the necessity for concerted research approaches in order to develop crops that are able to cope up with environmental challenges. Various abiotic stresses such as cold, heat, drought, salt, and heavy metal induce a variety of negative effects in plant growth, development and significantly decline yield and quality. Plant growth regulators or natural products of plants are reported to be effective to improve plant tolerance to several abiotic stresses. Gamma Amino Butyric Acid (GABA) is a non-protein amino acid involved in various metabolic processes, and partially protects plants from abiotic stress. GABA appears to impart partial protection to various abiotic stresses in most plants by increasing leaf turgor, increased osmolytes and reduced oxidative damage by stimulation of antioxidants. We have compiled various scientific reports on the role and mechanism of GABA in plants against coping with various environmental stresses. We have also described the emerging information about the metabolic and signaling roles of GABA which is being used to improve legume crop against abiotic stress.
\end{abstract}

Kumari Sita

sitas191@gmail.com

$\triangle$ Vaneet Kumar

veenumatri@gmail.com

1 Department of Botany, Panjab University, Chandigarh 160014, India

2 Department of Botany, S. L. Bawa DAV College, Batala 143501, India
Keywords Gamma Amino Butyric Acid (GABA) . Abiotic stress - Legumes - Climate change

\section{Introduction}

The worldwide population will reach 9.6 billion people, by 2050, and will face extreme challenges among which attaining food security is a high-priority issue (Stagnari et al. 2017). According to FAO (2020), even before COVID-19, 135 million people globally were already struggling with severe food insecurity and the present scenario creates a situation of "a crisis within a crisis". Food security is also a high-priority issue for achieving sustainable development goal 2 i.e. zero hunger (UN 2019). The Worldwide Global Nutrition Report (2020), highlighted that maximum countries of the world including India have large number of children who are stunted due of nutritional deficiencies. To meet these challenges, a policy framework needs to be developed in which the sustainability of production/consumption patterns becomes central. In this context, food legumes and legume-inclusive production systems can play important roles by delivering multiple services in line with sustainability principles (Stagnari et al. 2017).

Indeed, legumes play a central role; at food level system, both for human and animal consumption, as a source of plant protein and with an increasingly importance in improving human's health. At production level, their capacity to fix atmospheric nitrogen makes them potentially and extremely suitable for inclusion in low-input cropping systems and owing to their role in alleviating greenhouse gases emission (Jensen et al. 2010; Westhoek et al. 2011). At cropping system level, as diversification crops in agroecosystems based on some major crop species, 
breaking the cycles of pests and diseases and contributing to balance the deficiency in plant protein production in many areas of the world (Stagnari et al. 2017). Legumes rank third in world crop production, after cereals and oilseeds and the second most important crop family, with nearly 20,000 species (Sita et al. 2017). Grain legumes are cultivated in different seasons, namely cool-season food legumes such as broad bean (Vicia faba), lentil (Lens culinaris Medik.), lupin (Lupinus L.), dry pea (Pisum sativum), chickpea (Cicer arietinum), grass pea (Lathyrus sativus) and common vetch (Vicia sativa) and warm/tropical-season food legumes are pigeon pea (Cajanus cajan), cowpea (Vigna unguiculata), mungbean (Vigna radiata), common bean (Phaseolus sp.) and urd bean (Vigna mungo), which are mostly grown in hot and humid environments (Vadez et al. 2012; Sita et al. 2017). Legume cropping is expanding to warmer regions, and suitable for legume integration into annual cropping systems (Stagnari et al. 2017). Subsequently, in cropping systems legumes play a significant role in ensuring food security for rising population (Foyer et al. 2016; Considine et al. 2017; Liu et al. 2019).

The influence of change in climate and associated factors (biotic and abiotic) affects the crop production and yield, by declining average yields of major crop plants around the world (Chaves et al. 2003; Yadav et al. 2015). The increasing unpredictability of climate change certainly exaggerates these abiotic stresses, in turn affecting huge damage to the agricultural world. As a result, precise environmental adaptation strategies on target plants were developed (Beebe et al. 2011). One of the most noteworthy plant adaptation strategies to abiotic stresses was to activate multiple complex responses that involved physiological, biochemical, cellular, and molecular processes with many different pathways at the whole-plant level (Atkinson and Urwin 2012). According to Nayyar et al. (2014) one of the simplest and practical means to impart heat tolerance in heat sensitive crop species involves employing biomolecules with protective roles in stressed plants. Amino acids especially non-protein compounds such as GABA are reported to play a substantial role in functioning of plant, exclusively under stressful circumstances, due to their significant role in plant osmoregulation, antioxidant defense, and act as signaling molecule (Nayyar et al. 2014; Priya et al. 2019). Considering the significant role played by the GABA against different abiotic stresses and improving the plant varieties, the present review compiles information related to this topic focusing on legumes.

\section{GABA}

GABA (Gamma Amino Butyric Acid) a well-recognized ubiquitous non protein amino acid was reported in potato tuber (Thompson et al. 1953) and after 1 year later it was identified in mammalian brain (Awapara et al. 1950). It acts as endogenous signaling molecule in plant growth and development and rapidly accumulates in plant tissue in response to several abiotic and biotic stresses (Roberts 2007). Being an essential intermediate of nitrogen metabolism and amino acid biosynthesis, it plays a key role in primary and secondary metabolite synthesis (Ramos-Ruiz et al. 2019). Numerous research studies revealed that GABA is involved in facilitating tolerance to various abiotic stresses such as: temperature (Nayyar et al. 2014; Priya et al. 2019), water scarcity (Yong et al. 2017), salinity (Cheng et al. 2018), low light and nitrogen starvation (Kinnersley and Lin 2000). It stimulates plant growth and alleviates stress via up-regulating antioxidant defense system; therefore, it contributes to enhancement of shelf life due to higher antioxidants activity and quality of crop during storage (Ramos-Ruiz et al. 2019). The content of GABA depends upon the plant growth and developmental stage, and level of concentration significantly increased during germination of seeds (Kim et al. 2013). The functioning of GABA in plants has attracted new attentions in last decade following the findings that intracellular and extracellular GABA concentrations increase quickly in response to a variety of stresses (Shelp et al. 2017). Generally, level of GABA in plant tissue are ranged between 0.03 and $2.0 \mu \mathrm{molg}^{-1}$ (fresh weight), however it increased many folds in response to abiotic stress (Bhattacharya et al. 2018).

\section{Biosynthesis of GABA}

Generally, GABA is synthesized in vivo by a metabolic pathway known as GABA shunt pathway, firstly observed in plants (Steward 1949). GABA shunt functions for synthesis as well as maintenance of optimum GABA levels. It comprises of three main reactions catalyzed by cytosolic enzyme glutamate decarboxylase (GAD, E.C.4.1.1.15) mitochondrial enzymes GABA-T (E.C.2.6.1.19) and SSADH (succinic semi-aldehyde dehydrogenase) (E.C.1.2.2.16). Primarily, GAD (glutamic acid decarboxylase) catalyzes a-decarboxylation of glutamate to GABA in an irreversible reaction which is the rate limiting step in GABA synthesis (Shelp et al. 1995; Rashmi et al. 2018). GABA at this point converted to succinic semi aldehyde (SSA) by GABA-T and further SSADH converts SSA to succinate as described in Fig. 1 (Bown and Shelp 1997). 


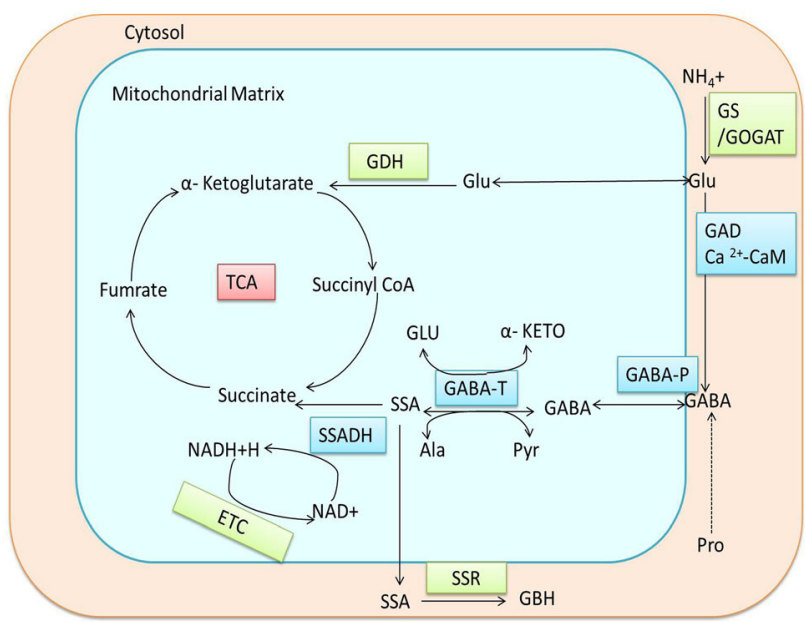

Fig. 1 Biosynthesis of GABA in plants by GABA shunt. Abbreviation's: (GAD) Glutamate decarboxylase; $\mathrm{Ca} 2+$ Calmodulin; (GABA-P) GABA permease; (GABA-T) GABA transaminase; $(\alpha-$ $\mathrm{KG}) \alpha$-ketoglutarate; (Pyr) pyruvate; (SSADH) succinic semialdehyde dehydrogenase; (ETC) electron transport chain; (SSA) succinic semialdehyde; (GBH) $\gamma$-hydroxybutyric acid; (SSR) succinic semialdehyde reductase. (Figure modified from Ramos-Ruiz et al. 2019)

GABA-T is dependent on pyruvate and glyoxylate as amino acceptors, thus producing alanine and glycine, correspondingly (Clark et al. 2009). In plants GABA-T favors 2-oxoglutarate over pyruvate and hence recovering glutamate for GAD reaction reported in tomato fruit. Therefore, succinate produced by SSADH may enter TCA cycle either can take part in electron transport chain in mitochondria as an electron donor (Akihiro et al. 2008). Yet, it is also observed in plants, animals, and E. coli that SSA can be changed to gamma-hydroxybutyrate (GHB) through a GHB dehydrogenase (GHBDH) (Rashmi et al. 2018). Apart from SSADH, all other enzymes of GABA biosynthetic pathway have been reported in multiple forms in plants; though, their number varies among species (Shelp et al. 2012; Trobacher et al. 2013). In plants, it is prominent that GAD has a calmodulin (CaM)-binding domain facilitating in vitro activity at $\mathrm{pH} 7.0-7.5$ to be turned-on by the $\mathrm{Ca}^{2+} / \mathrm{CaM}$ complex, whereas, at acidic $\mathrm{pH}, \mathrm{GAD}$ activity is least affected by $\mathrm{Ca}^{2+} / \mathrm{CaM}$ and has a sharp $\mathrm{pH}$ optimum at 5.8 (Rashmi et al. 2018; Priya et al. 2019). Alternate pathways of GABA synthesis from putrescine and other polyamines have also been reported. Here, gamma-amino butyraldehyde, an intermediary from polyamine degradation reaction through combined activities of diamine oxidase (DAO, E.C. 1.4.3.6) and 4-aminobutyraldehyde dehydrogenase (ABALDH), leads to the synthesis of GABA (Shelp et al. 2012).

\section{Role of GABA to mitigate abiotic stresses}

Abiotic stress increases GABA accumulation through two different mechanisms. Abiotic stress causes metabolic and a mechanical disruption that leads to cytosolic acidification induces an acidic $\mathrm{pH}$ dependent glutamate decarboxylase activation and GABA synthesis. Mei et al. (2016) reported a substantial decrease in cytosolic $\mathrm{pH}$ in waterlogged soils which have hypoxia condition and this stress enhanced the accumulation of GABA. The other mechanism is calcium dependent synthesis of GABA in various abiotic stresses such as heat, cold, salinity, drought and slight environmental fluctuations. These stresses quickly raise the cellular levels of $\mathrm{Ca}^{2+}$ and increased cytosolic $\mathrm{Ca}^{2+}$ stimulates calmodulin-dependent glutamate decarboxylase activity and GABA synthesis (Bhattacharya et al. 2018).

The abiotic stress tolerance of plants enhanced by the positive regulation of GABA shunt and associated pathways, whereas, endogenous GABA levels were enhanced by the exogenous application of GABA (Bao et al. 2015). Various studies reported on crop plants such as lentils (lens culinaris Medik.), melon (Cucumis melo L.), rice (Oryza sativa), and wheat (Triticum aestivum) shows that the exogenous application of GABA efficiently alleviated germination or growth inhibition caused by unfavorable environmental conditions such as extreme temperatures (Nayyar et al. 2014), drought (Li et al. 2016a), salt (Kumar et al. 2017), light or oxygen stress (Ramos-Ruiz et al. 2019). Exogenous GABA application in plants during stress condition, enhanced photosynthetic capacity, enhanced anti-oxidative enzymes, subsequently decrease in content of malondialdehyde (MDA), ROS accumulation, a marker for oxidative stress (Li et al. 2016b; Jia et al. 2017), and ultimately maintains the membrane integrity of GABA treated plants (Aghdam et al. 2015). Figure 2 summarizes the different mechanisms by which GABA helps plant to overcome various abiotic stresses.

\section{GABA and heat stress}

GABA accumulation during heat stress occurs through calcium-induced activation of enzyme glutamate decarboxylase, as observed in Arabidopsis plants (Locy et al. 2000; Priya et al. 2019). Exogenous application of GABA to heat stressed plant considerably improves growth as well as survival by reducing the membrane damage, improved cellular viability, chlorophyll content, and photochemical efficiency of plant leaves (Nayyar et al. 2014). Application of GABA improved the activities of CAT, SOD, APX and glutathione reductase in rice leaves, after short term heat stress indicating; defending role against heat persuaded 
Fig. 2 Role of GABA to mitigate the various abiotic stresses in plants

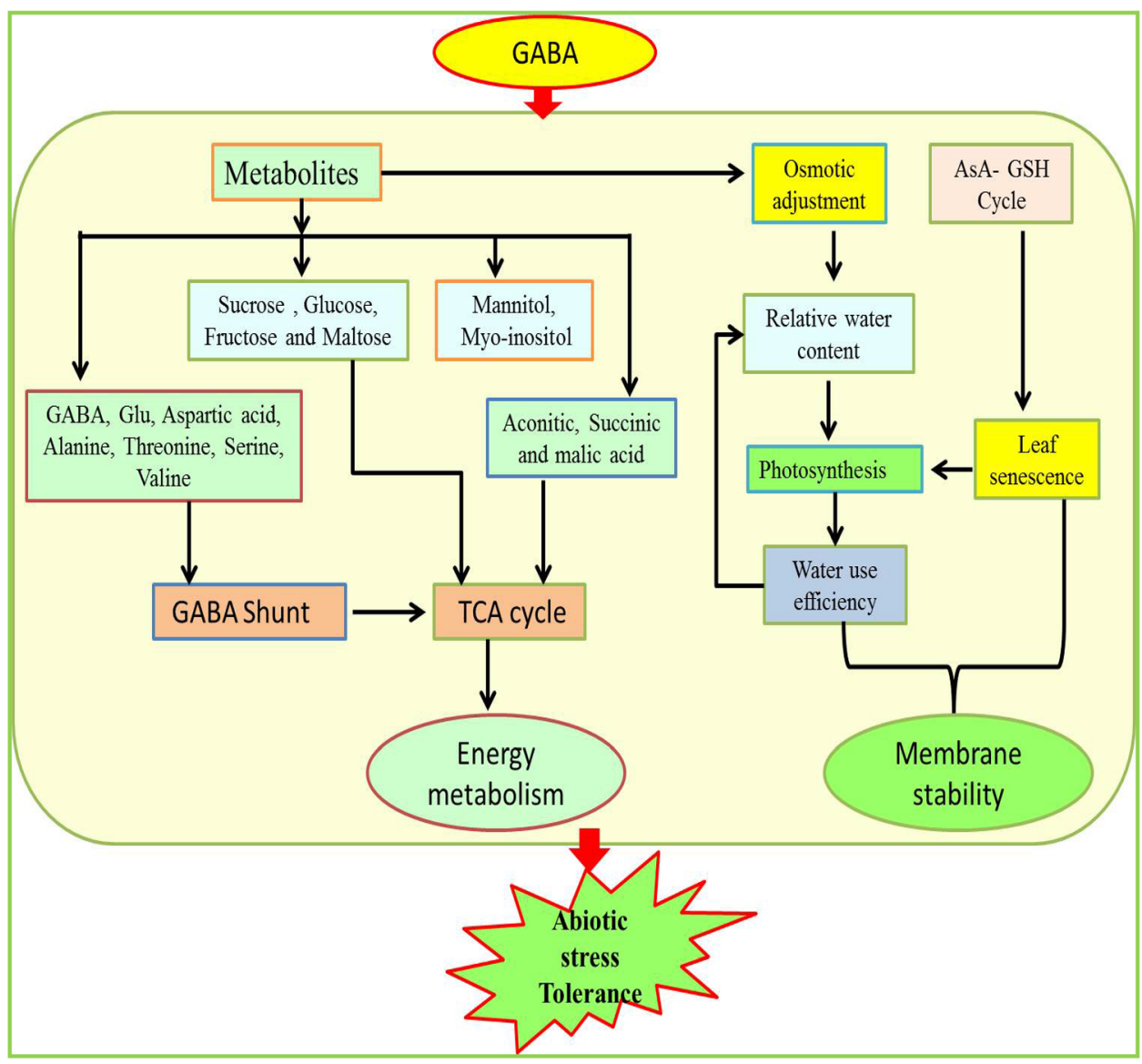

ROS (Nayyar et al. 2014). Similarly, Bor et al. (2009) reported that a short interval of heat shock also increased the endogenous content of GABA in cow pea and Sesamum indicum plants. Various physiological, morphological, and biochemical parameters were also improved with the application of GABA under elevated temperature stress legume plants (Ali et al. 2019).

Mungbean (Vigna radiata L.), a summer-season food legume rich in proteins, vitamins and minerals, has temperature optimum of about $35^{\circ} \mathrm{C} / 25^{\circ} \mathrm{C}$ (day/night). Temperature above $>36 / 28^{\circ} \mathrm{C}$ (day/night temperature) causes high temperature stress to the mungbean plants during reproductive period, causes severe losses such as loss of flowers, poor pod set, and reduced pod size, pod and seed numbers and seed yield (Kaur et al. 2015). In India, Panjab University Chandigarh Priya et al. (2019), raised mungbean plants in outdoor natural environment (29.3/ $16.1 \pm 1{ }^{\circ} \mathrm{C}$ as mean day/night temperature, $1350-1550 \mu \mathrm{mol} \mathrm{m}^{-2} \mathrm{~s}^{-1}$ light intensity, $60-65 \%$ as mean relative humidity) till the initiation of reproductive phase. Afterwards imposed a two dissimilar temperature treatments control $\left(35 / 23{ }^{\circ} \mathrm{C}\right)$ and heat stress $\left(45 / 28{ }^{\circ} \mathrm{C}\right)$ at, about $800 \mu \mathrm{mol} \mathrm{m} \mathrm{m}^{-2} \mathrm{~s}^{-1}$ light intensity and $65-70 \%$ as mean relative humidity, till pod maturity. The endogenous
GABA content levels declined $49-60 \%$ in elevated temperature stressed leaf and anther samples respectively, and to a much lesser degree in the plants, exogenously supplemented with $1 \mathrm{mM}$ GABA. Furthermore, exogenously GABA treatment considerably enhanced the reproductive function (pollen germination, pollen viability, stigma receptivity and ovule viability), less injury to membrane, photosynthetic machinery, carbon assimilation such as sucrose synthesis and its utilization and augmented accumulation of osmolytes as compared to control (untreated GABA) plants. The GABA application also produced a greater number of pods (28\%), higher seed weight $(27 \%)$ per plant as compared to controls without GABA application. The study revealed that GABA supplementation is beneficial for developmental and functional aspects of flowers, related to reproductive function (Priya et al. 2019). Very fewer studies are reported in this aspect, however in legumes there is only one study reported on this aspect till date. Therefore, more such studies are required to increase the intracellular GABA can induce the expression of signaling and metabolism associated genes involved in the regulation of plant growth and plant vigor and improved stress tolerance. 


\section{GABA and drought stress}

Drought stress is one of the most severe issues that deleteriously affect plant growth and development of plant species around the world (Sehgal et al. 2018). Food crops, such as grain legumes, which are commonly grown in rainfed areas, are more prone to drought stress; The worldwide climate models have predicted continuous variability and rise in the intensity and frequency of drought incidents (Rosenzweig and Hillel 2005; Sehgal et al. 2019). The deficiency of accessible water restrains plant productivity owing to drought-induced cellular membrane damage, nutritional imbalance, and metabolic disorders (Zhao et al. 2020).

Yong et al. (2017), stated that the exogenous application of GABA is an effective approach to improve the drought stress form plants. The exogenous application of GABA efficiently improved drought induced injury in white clover leaves, as reflected by significantly more relative water content, reduced electrolyte leakage, lipid peroxidation (LPO) content, and leaf wilting. GABA application substantially enhances the drought tolerance via reducing the lipid peroxidation content and enhancing the photosynthetic pigments and mitochondrial activity in black pepper (Vijayakumari and Puthur 2016). The exogenous application of GABA also enhanced drought resistance in creeping bent grass accompanying with the augmented accumulation of amino acids, organic acids, and other osmotic substances related to secondary metabolism ( $\mathrm{Li}$ et al. 2016a). Furthermore, exogenous application of GABA in drought induced white clover leaf rises in GABA transaminase and alpha ketone glutarate dehydrogenase activities, however inhibited glutamate decarboxylase activity under both control and drought conditions, leading to a rise in endogenous glutamate (Glu) and GABA concentration (Yong et al. 2017). Mekonnen et al. (2016), reported that the decline of GABA concentration closely in relation to a reduced resistance to drought in Arabidopsis mutant by the knock-out of glutamate decarboxylase gene.

GABA concentrations are reported higher in legume nodules of common bean (Antoniw and Sprent 1978), soyabean (Serraj et al. 1998). Serraj et al. (1998), reported that under drought stress, the accumulation of GABA occurs in all parts of soybean plant tissues, whereas it was more pronounced in nodules as compared to leaf and root tissue. GAD activities are more in leaf and root tissue as compared to nodules. This is due the decline in oxygen permeability of nodules and oxygen deficiency inside the nodule stimulates the GAD activities. Therefore, exogenous application of GABA may increase the endogenous level of GABA in drought stressed plants and could improve the drought tolerance. The studies on exogenous application of GABA in drought stressed plants especially in legumes are not reported in detail and studies are required for food security and better yield production of crop legumes grown in water deficit area.

\section{GABA and cold stress}

The temperature on the earth's surface is very diverse, changing during the seasons as well as during the day and night. In plants cold and chilling temperature normally ranges from 0 to $15{ }^{\circ} \mathrm{C}$ (Malekzadeh et al. 2012). Low temperature stress deleteriously affects the plant growth and development and can reduce the yield up to $15-20 \%$ (Chaturvedi et al. 2009).

Yang et al. (2011) studies described that GABA application significantly inhibited the cold /low temperature injury and enhanced the anti-oxidative enzyme activities, such as superoxide dismutase, catalase, ascorbate peroxidase, glutathione peroxidase, glutathione S-transferase, mono-dehydro ascorbate reductase and dehydro-ascorbate reductase in peach fruit. The exogenous GABA application also enhanced the content of adenosine triphosphate and adenosine diphosphate, however declined the adenosine monophosphate content, which resulted in a higher level of energy charge in GABA treated fruit. There are some other reports on role of GABA to mitigating cold / chilling stress such as in peach fruit (Shang et al. 2011; Bustamante et al. 2016), wheat seedlings (Malekzadeh et al. 2012), zucchini (Palma et al. 2019), cucumber (Malekzadeh et al. 2017), banana (Wang et al. 2016), litchi (Zhou et al. 2019) fruits, and tomato seedlings (Malekzadeh et al. 2014). However, the roles of exogenous GABA application in cold stressed legume are not reported yet.

\section{GABA and salt stress}

Salinity in soil mainly due to $\mathrm{NaCl}$ causes substantial abiotic stress to the plants and affects both irrigated and non-irrigated land area. On a worldwide scale, around $20 \%$ of cultivated land and almost 50\% of irrigated land is affected by high salinity. It has been expected that approximately $30 \%$ of the available land will be lost within the next 25 years and up to $50 \%$ will be lost by 2050 due to soil salinity (Wang et al. 2017). Taking into consideration the projected increasing world population and food demand, finding ways to develop crop tolerance to salt stress constraints is a serious issue for further improving agricultural production and enhancing global food security (Sheteiwy et al. 2019). Exogenous GABA application declines the accumulation of Reactive Oxygen Species (ROS) by increasing the various anti-oxidative enzymes 
and osmolytes, help sustain cell morphology, and increase the function of cells under salt stress (Wang et al. 2017).

Kumar et al. (2017), conducted an experiment to examine the effect of GABA on morphological characters and yield attributes of salt stressed blackgarm (Vigna mungo). The results revealed that the plant height, plant dry weight, and pod number per plant were significantly higher in all the stages $30,45,60$ DAS (days after sowing) by the treatment of $(\mathrm{NaCl} 50 \mathrm{mM}+\mathrm{GABA} 70 \mathrm{mM})$ compared to all control treatment of $\mathrm{NaCl}$ i.e. $100 \mathrm{mM}$. Whereas maximum seed yield $3.9 \mathrm{~g}$ was recorded in $(\mathrm{NaCl} 50 \mathrm{mM}+$ GABA $70 \mathrm{mM}$ ) treatment, which was significantly superior over all other treatment and control was recorded.

Al-Quraan and Al-Omari (2017), studied the response of two lentil cultivars (Lens culinaris Medik.) (Jordan 1 and Jordan 2) to $\mathrm{NaCl}$, mannitol, sorbitol, and $\mathrm{H}_{2} \mathrm{O}_{2}$ via the characterization of seed germination, accumulation of ROS, and $\gamma$-aminobutyric acid (GABA) level. GABA application significantly enhances the malondialdehyde (MDA) content in the two lentil cultivars in all the treatments. The lentil cultivars Jordan 1 showed the maximum germination percentages, whereas, Jordan 2 seedlings showed the highest GABA levels and MDA accumulation under salt, osmotic, and oxidative stresses, respectively, compared with Jordan 1 seedlings under the same treatments. The results showed that GABA shunt is a key signaling and metabolic pathway that allows adaptation of lentil seedlings to salt, osmotic, and oxidative stresses. The results revealed that GABA shunt is an important signaling and metabolic pathway that allows adaptation of lentil seedlings to salt, osmotic, and oxidative stresses. Sheteiwy et al. (2019), conducted an experiment on rice (Oryza sativa L.) by priming of $\gamma$-aminobutyric acid (GABA) at $0.5 \mathrm{mM}$ on seed germination under osmotic stress (OS) induced by polyethylene glycol (30 g/L PEG 6000); and salinity stress $(\mathrm{S}, 150 \mathrm{mM} \mathrm{NaCl})$ and their combination $(\mathrm{OS}+\mathrm{S})$. Priming with GABA significantly alleviated the negative effects of OS, $\mathrm{S}$ and $\mathrm{OS}+\mathrm{S}$ on seed germination and seedling growth. The GABA primed-plants, salinity stress alone resulted in a noticeable increase in the expression level of Calcineurin B-like Protein-interacting protein Kinases (CIPKs) genes such as OsCIPKO1, OsCIPKO3, OsCIPKO8 and OsCIPK15, and osmotic stress alone resulted in obvious increase in the expression of OsCIPKO2, OsCIPKO7 and OsCIPK09; and OS + S resulted in a significant up-regulation of $O s C I P K 12$ and OsCIPK17. The results showed that salinity, osmotic stresses and their combination induced changes in cell ultra-morphology and cell cycle progression resulting in prolonged cell cycle development duration and inhibitory effects on rice seedlings growth. These findings recommended that the high tolerance to $\mathrm{OS}+\mathrm{S}$ is closely associated with the capability of GABA priming to control the reactive oxygen species (ROS) level by inducing antioxidant enzymes, secondary metabolism and their transcription level. This knowledge provides new evidence for better understanding molecular mechanisms of GABAregulating salinity and osmotic-combined stress tolerance during rice seed germination and development. However, in legumes no such studies are reported yet on this aspect and need to be explored.

\section{GABA and heavy metal stress}

Rapid rate of industrialization, urbanization, intensive agriculture, and mining has been triggering widespread contamination of our soil and aquifer resources with heavy metals (Rai et al. 2019). Soils contaminated by heavy metals ( $\mathrm{Zn}, \mathrm{Ni}, \mathrm{Fe}, \mathrm{Co}, \mathrm{Cr}, \mathrm{Cd}, \mathrm{Pb}, \mathrm{As}$ and $\mathrm{Hg}$ ) from agricultural activities have raised serious concern in recent decades regarding potential threat to human health through the direct intake, bioaccumulation through food chain, and their influences on ecological system (Naveedullah et al. 2013). Although some of metal elements are essential micronutrients responsible for normal growth and metabolism of plants (Mani and Sankaranarayanan 2018), but excessive metal accumulation in plant tissues impair either directly or indirectly several biochemical, physiological, and morphological functions in plants and in turns interferes with crop productivity (Shahid et al. 2015).

Song et al. (2010), reported that GABA application mitigates the oxidative injury affected by the $\mathrm{H}^{+}$and aluminum toxicity in barley seedlings by activating anti-oxidative enzymes as a defense response and declining the raised levels of carbonylated proteins caused by ROS. The exogenous application of GABA decreases the protein and lipid injury, signifying that GABA is critical for cellular stress response to $\mathrm{H}^{+}$and aluminum in plants. The studies on the applications of GABA to mitigate the effects of metal stress on legumes are not reported yet. However, the role of GABA to mitigates the effects of metal stress in many other crop species are reported such as, cadmium toxicity in maize (Seifikalhor et al. 2020), chromium toxicity in brassica (Mahmud et al. 2017) and arsenite toxicity in rice (Kumar et al. 2019) are reported.

\section{Health benefits of GABA and legumes}

GABA, a non-proteinogenic amino acid is not only important for mitigating different kinds of stress in plants; however, it has various health benefits including reduction of hypertension (Yoshimura et al. 2010), inhibition of chronic diseases associated with alcohol (Oh et al. 2003), prevention of cancer cell proliferation (Dilis and 
Trichopoulou 2009), and modulation of blood cholesterol levels (Roohinejad et al. 2009). Legumes are a good potential source for GABA production due to their high amounts of proteins (Nikmaram et al. 2017). L-glutamic acid, the substrate for GABA synthesis, is one of the most abundant amino acids found in pulses as reported in faba bean (Khalil and Mansour 1995) and mungbeans (Mubarak 2005).

The previous studies have reported various associations between the consumption of pulses and health benefits; including the decline of the risk of chronic diseases e.g. obesity, diabetes, coronary heart disease, stroke, hypertension, and some types of cancer. The mechanism by which legumes may prevent against diseases could involve the action of macronutrients including resistant starch and non-nutrient bioactive compounds such as phytates (Mathers, 2002). The consumption of food with high GABA concentration was found to reduce the elevation of blood pressure and cholesterol in experimental animals (Abe et al., 1995; Inoue et al., 2003; Hayakawa et al. 2004). Various factors alter the content of GABA in legumes such as; type of cultivar, environmental stress during plant growth, and the processing method of the seeds such as soaking, cooking, germination or fermentation (Nikmaram et al. 2017). In present situation we need to development of new functional foods from legumes with enhanced GABA levels targeted at the whole population or for particular groups at risk of chronic diseases also.

\section{Conclusion and future perspectives}

The exogenous application of GABA enhances the endogenous GABA levels and the tolerance of plants, and improved abiotic stress tolerance via positive regulation of the GABA shunt and associated pathways. GABA application in stressed plants increases the GABA shunt activity, increased photosynthetic efficiency, endogenous GABA, anti-oxidative enzymes, and decline in MDA content an oxidative stress marker, reactive oxygen species, and finally the membrane integrity of the treated plants was retained.

Furthermore, various nutritional traits in legumes were highly influenced with environmental stresses and an effect of GABA on mineral acquisition in plants was reported in experiments with duckweed (Lemna minor L.). They reported that plants treated with $10 \mathrm{mM}$ of GABA had higher levels of macronutrients and higher levels of $\mathrm{Mn}, \mathrm{Zn}$ and $B$ than untreated plants. Therefore, application of GABA could be a promising strategy for increasing the nutrient content of legume crops from a biofortification perspective. In conclusion, it is suggested that GABA plays a positive role in physiological modulation when plants suffer from unfavorable environmental stresses and the manipulation of GABA levels could be a potential method in enhancing stress resistance in plants. This review article highlighted various positive impacts of GABA on plants together with the ability to induce stress tolerance. This comprehensive report about the synthesis and stress mitigation role of GABA could be considered as a tool to improve the quantity and quality of agricultural crops especially legumes and help to achieving sustainable development goal number 2 i.e. zero hunger.

Authors' contributions KS conceived the concept and VK supported the idea. KS collected all the literature and composed all the information and wrote the article. VK extensively edited the article and $\mathrm{HN}$ give valuable suggestions.

Funding This work is not supported by any funding agency.

\section{Compliance with ethical standards}

Conflict of interest The authors declare that there are no conflicts of interest or any competing interests regarding the publication of this paper.

\section{References}

Abe, Y., Umemura, S., Sugimoto, K. I., Hirawa, N., Kato, Y., Yokoyama, N., et al. (1995). Effect of green tea rich in $\gamma$ aminobutyric acid on blood pressure of Dahl salt-sensitive rats. American Journal of Hypertension, 8, 74-79.

Aghdam, M. S., Naderi, R., Sarcheshmeh, M. A. A., \& Babalar, M. (2015). Amelioration of postharvest chilling injury in anthurium cut flowers by $\gamma$-aminobutyric acid (GABA) treatments. Postharvest Biology and Technology, 110, 70-76.

Akihiro, T., Koike, S., Tani, R., Tominaga, T., Watanabe, S., Iijima, Y., et al. (2008). Biochemical mechanism on GABA accumulation during fruit development in tomato. Plant and Cell Physiology, 49, 1378-1389.

Ali, S., Rizwan, M., Arif, M. S., Ahmad, R., Hasanuzzaman, M., Ali, B.,Hussain, A. (2019). Approaches in enhancing thermotolerance in plants: An updated review. Journal of Plant Growth Regulation, 39, 456-480.

Al-Quraan, N. A., \& Al-Omari, H. A. (2017). GABA accumulation and oxidative damage responses to salt, osmotic and $\mathrm{H} 2 \mathrm{O} 2$ treatments in two lentil (Lens culinaris Medik) accessions. Plant Biosystems-An International Journal Dealing with all Aspects of Plant Biology, 151(1), 148-157.

Antoniw, L. D., \& Sprent, J. I. (1978). Primary metabolites of Phaseolus vulgaris nodules. Phytochemistry, 17, 675-678.

Atkinson, N. J., \& Urwin, P. E. (2012). The interaction of plant biotic and abiotic stresses: From genes to the field. Journal of Experimental Botany, 63, 3523-3543.

Awapara, J., Landua, A. J., Fuerst, R., \& Seale, B. (1950). Free gamma-amino butyric acid in brain. The Journal of biological chemistry, 187, 35-39.

Bao, H., Chen, X., Lv, S., Jiang, P., Feng, J., Fan, P., et al. (2015). Virus-induced gene silencing reveals control of reactive oxygen species accumulation and salt tolerance in tomato by $\gamma$ aminobutyric acid metabolic pathway. Plant, Cell and Environment, 38, 600-613. 
Beebe, S., Ramirez, J., Jarvis, A., Rao, I. M., Mosquera, G., Bueno, J. M., \& Blair, M. W. (2011). Genetic improvement of common beans and the challenges of climate change. Crop Adaptation to Climate Change, 356-369.

Bhattacharya, S., Khatri, A., Swanger, S. A., DiRaddo, J. O., Yi, F., Hansen, K. B., et al. (2018). Triheteromeric GluN1/GluN2A/ GluN2C NMDARs with unique single-channel properties are the dominant receptor population in cerebellar granule cells. $\mathrm{Neu}$ ron, 99, 315-328.

Bor, M., Seckin, B., Ozgur, R. E., Yılmaz, O., Ozdemir, F., \& Turkan, I. (2009). Comparative effects of drought, salt, heavy metal and heat stresses on gamma-aminobutryric acid levels of sesame (Sesamum indicum L.). Acta Physiologiae Plantarum, 31, $655-659$.

Bown, A. W., \& Shelp, B. J. (1997). The metabolism and functions of [gamma]-aminobutyric acid. Plant Physiology, 115, 1.

Bustamante, C. A., Monti, L. L., Gabilondo, J., Scossa, F., Valentini, G., Budde, C. O., et al. (2016). Differential metabolic rearrangements after cold storage are correlated with chilling injury resistance of peach fruits. Frontiers in Plant Science, 7, 1478.

Chaturvedi, S. K., Mishra, D. K., Vyas, P., \& Mishra, N. (2009). Breeding for cold tolerance in chickpea. Trends in Biosciences, 2, $1-4$.

Chaves, M. M., Maroco, J. P., \& Pereira, J. S. (2003). Understanding plant responses to drought-from genes to the whole plant. Functional Plant Biology, 30, 239-264.

Cheng, B., Li, Z., Liang, L., Cao, Y., Zeng, W., Zhang, X., et al. (2018). The $\gamma$-aminobutyric acid (GABA) alleviates salt stress damage during seeds germination of white clover associated with $\mathrm{Na}+/ \mathrm{K}+$ transportation, dehydrins accumulation, and stress-related genes expression in white clover. International Journal of Molecular Sciences, 19, 2520.

Clark, S., Di, M., Leo, R., Dhanoa, P. K., Van Cauwenberghe, O. R., Mullen, R. T., et al. (2009). Biochemical characterization, mitochondrial localization, expression, and potential functions for an Arabidopsis $\gamma$-aminobutyrate transaminase that utilizes both pyruvate and glyoxylate. Journal of Experimental Botany, 60, 1743-1757.

Considine, M. J., Siddique, K. H., \& Foyer, C. H. (2017). Nature's pulse power: Legumes, food security and climate change. Journal of Experimental Botany, 68, 1815-1818.

Dilis, V., \& Trichopoulou, A. (2009). Nutritional and health properties of pulses. Mediterranean Journal of Nutrition and Metabolism, 1, 149-157.

FAO. (2020). http://www.fao.org/news/story/en/item/1269721/icode/. Retrieved 15 Sept 2020.

Foyer, C. H., Lam, H. M., Nguyen, H. T., Siddique, K. H., Varshney, R. K., Colmer, T. D., et al. (2016). Neglecting legumes has compromised human health and sustainable food production. Nature Plants, 2, 1-10.

Hayakawa, K., Kimura, M., Kasaha, K., Matsumoto, K., Sansawa, H., \& Yamori, Y. (2004). Effect of a $\gamma$-aminobutyric acid-enriched dairy product on the blood pressure of spontaneously hypertensive and normotensive Wistar-Kyoto rats. British Journal of Nutrition, 92, 411-417.

Inoue, K., Shirai, T., Ochiai, H., Kasao, M., Hayakawa, K., Kimura, M., et al. (2003). Blood-pressure-lowering effect of a novel fermented milk containing $\gamma$-aminobutyric acid (GABA) in mild hypertensives. European Journal of Clinical Nutrition, 57, 490-495.

Jensen, E. S., Peoples, M. B., \& Hauggaard-Nielsen, H. (2010). Faba bean in cropping systems. Field Crops Research, 115, 203-216.

Jia, Y., Zou, D., Wang, J., Sha, H., Liu, H., Inayat, M. A., et al. (2017). Effects of $\gamma$-aminobutyric acid, glutamic acid, and calcium chloride on rice (Oryza sativa L.) under cold stress during the early vegetative stage. Journal of Plant Growth Regulation, 36, 240-253.

Kaur, R., Bains, T. S., Bindumadhava, H., \& Nayyar, H. (2015). Responses of mungbean (Vigna radiata L.) genotypes to heat stress: Effects on reproductive biology, leaf function and yield traits. Scientia Horticulturae, 197, 527-541.

Khalil, A. H., \& Mansour, E. H. (1995). The effect of cooking, autoclaving and germination on the nutritional quality of faba beans. Food Chemistry, 54, 177-182.

Kim, N. H., Kim, B. S., \& Hwang, B. K. (2013). Pepper arginine decarboxylase is required for polyamine and $\gamma$-aminobutyric acid signaling in cell death and defense response. Plant Physiology, 162, 2067-2083.

Kinnersley, A. M., \& Lin, F. (2000). Receptor modifiers indicate that 4-aminobutyric acid (GABA) is a potential modulator of ion transport in plants. Plant Growth Regulation, 32, 65-76.

Kumar, N., Gautam, A., Dubey, A. K., Ranjan, R., Pandey, A., Kumari, B., et al. (2019). GABA mediated reduction of arsenite toxicity in rice seedling through modulation of fatty acids, stress responsive amino acids and polyamines biosynthesis. Ecotoxicology and Environmental Safety, 173, 15-27.

Kumar, S., Jangde, S., Priya, T., \& Yashu, B. R. (2017). Effect of GABA on morphology, yield and yield attributes in Black Gram (Vigna mungo (L.) Hepper) under salt stress condition. International Journal of Pure Applied Biosciences, 5, 1223-1228.

Li, M. F., Guo, S. J., Yang, X. H., Meng, Q. W., \& Wei, X. J. (2016a). Exogenous gamma-aminobutyric acid increases salt tolerance of wheat by improving photosynthesis and enhancing activities of antioxidant enzymes. Biologia Plantarum, 60, 123-131.

Li, Z., Peng, Y., \& Huang, B. (2016b). Physiological effects of $\gamma$ aminobutyric acid application on improving heat and drought tolerance in creeping bentgrass. Journal of the American Society for Horticultural Science, 141, 76-84.

Liu, W., Min, X., \& Wang, Y. (2019). Genome-wide development of microRNA-based SSR markers in Medicago truncatula with their transferability analysis and utilization in related legume species. The Model Legume Medicago truncatula, 936-945.

Locy, R. D., Wu, S. J., Bisnette, J., Barger, T. W., McNabb, D., Zik, M., et al. (2000). The regulation of GABA accumulation by heat stress in Arabidopsis. In Joe H. Cherry, Robert D. Locy, \& Anna Rychter (Eds.), Plant tolerance to abiotic stresses in agriculture: Role of genetic engineering (pp. 39-52). Dordrecht: Springer.

Mahmud, J. A., Hasanuzzaman, M., Nahar, K., Rahman, A., Hossain, M. S., \& Fujita, M. (2017). $\gamma$-aminobutyric acid (GABA) confers chromium stress tolerance in Brassica juncea L. by modulating the antioxidant defense and glyoxalase systems. Ecotoxicology, 26, 675-690.

Malekzadeh, P., Khara, J., \& Haidari, R. (2012). Effect of exogenous Gama-aminobutyric acid on physiological tolerance of wheat seedlings exposed to chilling stress. Iranian Journal of Plant Physiology, 3, 611-617.

Malekzadeh, P., Khara, J., \& Heydari, R. (2014). Alleviating effects of exogenous Gamma-aminobutiric acid on tomato seedling under chilling stress. Physiology and Molecular Biology of Plants, 20, 133-137.

Malekzadeh, P., Khosravi-Nejad, F., Hatamnia, A. A., \& Mehr, R. S. (2017). Impact of postharvest exogenous $\gamma$-aminobutyric acid treatment on cucumber fruit in response to chilling tolerance. Physiology and Molecular Biology of Plants, 23, 827-836.

Mani, A., \& Sankaranarayanan, K. (2018). Heavy metal and mineral element-induced abiotic stress in rice plant. Developments, https://doi.org/10.5772/intechopen.76080.

Mathers, J. C. (2002). Pulses and carcinogenesis: Potential for the prevention of colon, breast and other cancers. British Journal of Nutrition, 88, 273-279. 
Mei, X., Chen, Y., Zhang, L., Fu, X., Wei, Q., Grierson, D., et al. (2016). Dual mechanisms regulating glutamate decarboxylases and accumulation of gamma-aminobutyric acid in tea (Camellia sinensis) leaves exposed to multiple stresses. Scientific Reports, 6, 23685.

Mekonnen, D. W., Flügge, U. I., \& Ludewig, F. (2016). Gammaaminobutyric acid depletion affects stomata closure and drought tolerance of Arabidopsis thaliana. Plant Science, 245, 25-34.

Mubarak, A. E. (2005). Nutritional composition and anti-nutritional factors of mung bean seeds (Phaseolus aureus) as affected by some home traditional processes. Food Chemistry, 89, 489-495.

Naveedullah, N., Hashmi, M. Z., Yu, Ch., Shen, H., Duan, D., Shen, Ch., et al. (2013). Risk assessment of heavy metals pollution in agricultural soils of siling reservoir watershed in Zhejiang province, China. Journal of Biomedical and Biotechnology, 2013, 1-10.

Nayyar, H., Kaur, R., Kaur, S., \& Singh, R. (2014). $\gamma$-Aminobutyric acid (GABA) imparts partial protection from heat stress injury to rice seedlings by improving leaf turgor and upregulating osmoprotectants and antioxidants. Journal of Plant Growth Regulation, 33, 408-419.

Nikmaram, N., Dar, B. N., Roohinejad, S., Koubaa, M., Barba, F. J., Greiner, R., et al. (2017). Recent advances in $\gamma$-aminobutyric acid (GABA) properties in pulses: An overview. Journal of the Science of Food and Agriculture, 97, 2681-2689.

Oh, S. H., Moon, Y. J., \& Oh, C. H. (2003). $\gamma$-Aminobutyric acid (GABA) content of selected uncooked foods. Preventive Nutrition and Food Science, 8, 75-78.

Palma, F., Carvajal, F., Jiménez-Muñoz, R., Pulido, A., Jamilena, M., \& Garrido, D. (2019). Exogenous $\gamma$-aminobutyric acid treatment improves the cold tolerance of zucchini fruit during postharvest storage. Plant Physiology and Biochemistry, 136, 188-195.

Priya, M., Sharma, L., Kaur, R., Bindumadhava, H., Nair, R. M., Siddique, K. H. M., et al. (2019). GABA ( $\gamma$-aminobutyric acid), as a thermo-protectant, to improve the reproductive function of heat-stressed mungbean plants. Scientific Reports, 9, 1-14.

Rai, P. K., Lee, S. S., Zhang, M., Tsang, Y. F., \& Kim, K. H. (2019). Heavy metals in food crops: Health risks, fate, mechanisms, and management. Environment International, 125, 365-385.

Ramos-Ruiz, R., Martinez, F., \& Knauf-Beiter, G. (2019). The effects of GABA in plants. Cogent Food and Agriculture, 5, 1670553.

Rashmi, D., Zanan, R., John, S., Khandagale, K., \& Nadaf, A. (2018). $\gamma$-aminobutyric acid (GABA): Biosynthesis, role, commercial production, and applications. Studies in Natural Products Chemistry Elsevier, 57, 413-452.

Roberts, M. R. (2007). Does GABA act as a signal in plants? Hints from molecular studies: hints from molecular studies. Plant Signaling Behavior, 2, 408-409.

Roohinejad, S., Omidizadeh, A., Mirhosseini, H., Rasti, B., Saari, N., Shuhaimi, M., Yusof, R. M., Meor Hussin, A. S., Hamid, A., \& Manap, M. Y. (2009). Effect of hypocholesterolemic properties of brown rice varieties containing different gamma amino butyric acid (GABA) levels on Sprague-Dawley male rats. Journal of Food Agriculture and Environment, 7(3\&4), 197-203.

Rosenzweig, C., \& Hillel, D. (2005). Global warming and agriculture. Perspectives in World Food and Agriculture, 2, 181-210.

Shahid, M., Khalid, S., Abbas, G., Shahid, N., Nadeem, M., Sabir, M., Aslam, M., \& Dumat, C. (2015). Heavy metal stress and crop productivity. In Crop production and global environmental issues (pp. 1-25). Cham: Springer.

Sehgal, A., Sita, K., Bhandari, K., Kumar, S., Kumar, J., Vara Prasad, P. V., et al. (2019). Influence of drought and heat stress, applied independently or in combination during seed development, on qualitative and quantitative aspects of seeds of lentil (Lens culinaris Medikus) genotypes, differing in drought sensitivity. Plant, Cell and Environment, 42, 198-211.

Sehgal, A., Sita, K., Siddique, K. H., Kumar, R., Bhogireddy, S., Varshney, R. K., et al. (2018). Drought or/and heat-stress effects on seed filling in food crops: Impacts on functional biochemistry, seed yields, and nutritional quality. Frontiers in Plant Science, 9, 1705 .

Seifikalhor, M., Aliniaeifard, S., Bernard, F., Seif, M., Latifi, M., Hassani, B., et al. (2020). $\gamma$-Aminobutyric acid confers cadmium tolerance in maize plants by concerted regulation of polyamine metabolism and antioxidant defense systems. Scientific Reports, 10, 1-18.

Serraj, R., Shelp, B. J., \& Sinclair, T. R. (1998). Accumulation of $\gamma$ aminobutyric acid in nodulated soybean in response to drought stress. Physiologia Plantarum, 102, 79-86.

Shang, H., Cao, S., Yang, Z., Cai, Y., \& Zheng, Y. (2011). Effect of exogenous $\gamma$-aminobutyric acid treatment on proline accumulation and chilling injury in peach fruit after long-term cold storage. Journal of Agricultural and Food Chemistry, 59, 1264-1268.

Shelp, B. J., Bown, A. W., \& Zarei, A. (2017). 4-Aminobutyrate (GABA): A metabolite and signal with practical significance. Botany, 95, 1015-1032.

Shelp, B. J., Mullen, R. T., \& Waller, J. C. (2012). Compartmentation of GABA metabolism raises intriguing questions. Trends in Plant Science, 17, 57-59.

Shelp, B. J., Walton, C. S., Snedden, W. A., Tuin, L. G., Oresnik, I. J., \& Layzell, D. B. (1995). GABA shunt in developing soybean seeds is associated with hypoxia. Physiologia Plantarum, 94, 219-228.

Sheteiwy, M. S., Shao, H., Qi, W., Hamoud, Y. A., Shaghaleh, H., Khan, N. U., et al. (2019). GABA-alleviated oxidative injury induced by salinity, osmotic stress and their combination by regulating cellular and molecular signals in rice. International Journal of Molecular Sciences, 20, 5709.

Sita, K., Sehgal, A., Hanumantha Rao, B., Nair, R. M., Vara Prasad, P. V., Kumar, S., et al. (2017). Food legumes and rising temperatures: Effects, adaptive functional mechanisms specific to reproductive growth stage and strategies to improve heat tolerance. Frontiers in Plant Science, 8, 1658.

Song, H., Xu, X., Wang, H., Wang, H., \& Tao, Y. (2010). Exogenous $\gamma$-aminobutyric acid alleviates oxidative damage caused by aluminium and proton stresses on barley seedlings. Journal of the Science of Food and Agriculture, 90(9), 1410-1416.

Stagnari, F., Maggio, A., Galieni, A., \& Pisante, M. (2017). Multiple benefits of legumes for agriculture sustainability: An overview. Chemical and Biological Technologies in Agriculture, 4, 2.

Steward, F. C. (1949). $\gamma$-Aminobutyric acid: A constituent of the potato tuber? Science, 110, 439-440.

Thompson, J. F., Pollard, J. K., \& Steward, F. C. (1953). Investigations of nitrogen compounds and nitrogen metabolism in plants. III. Gamma-aminobutyric acid in plants, with special reference to the potato tuber and a new procedure for isolating amino acids other than alpha-amino acids. Plant Physiology, 28, 401-414.

Trobacher, C. P., Clark, S. M., Bozzo, G. G., Mullen, R. T., DeEll, J. R., \& Shelp, B. J. (2013). Catabolism of GABA in apple fruit: Subcellular localization and biochemical characterization of two $\gamma$-aminobutyrate transaminases. Postharvest Biology and Technology, 75, 106-113.

UN. (2019). The Sustainable Development Goals Report 2019. https://unstats.un.org/sdgs/report/2019/The-Sustainable-Develop ment-Goals-Report-2019.pdf. Retrieved 7 July 2020.

Vadez, V., Berger, J. D., Warkentin, T., Asseng, S., Ratnakumar, P., Rao, K. P. C., et al. (2012). Adaptation of grain legumes to climate change: A review. Agronomy for Sustainable Development, 32, 31-44. 
Vijayakumari, K., \& Puthur, J. T. (2016). $\gamma$-Aminobutyric acid (GABA) priming enhances the osmotic stress tolerance in Piper nigrum Linn. plants subjected to PEG-induced stress. Plant Growth Regulation, 78, 57-67.

Wang, Y., Gu, W., Meng, Y., Xie, T., Li, L., Li, J., et al. (2017). $\gamma$ Aminobutyric acid imparts partial protection from salt stress injury to maize seedlings by improving photosynthesis and upregulating osmoprotectants and antioxidants. Scientific Reports, 7, 43609.

Wang, Y., Luo, Z., Mao, L., \& Ying, T. (2016). Contribution of polyamines metabolism and GABA shunt to chilling tolerance induced by nitric oxide in cold-stored banana fruit. Food Chemistry, 197, 333-339.

Westhoek, H., Rood, T., vanden Berg, M., Janse, J., Nijdam, D., Reudink, M., \& Stehfest, E. (2011). The protein puzzles. The consumption and production of meat, dairy and fish in the European Union. Netherlands Environmental Assessment Agency (PBL)

Worldwide Global Nutrition Report. (2020). https://globalnutritionre port.org/reports/global-nutrition-report-2020/. Retrieved 12 May 2020.

Yadav, S. S., Hunter, D., Redden, B., Nang, M., Yadava, D. K., \& Habibi, A. B. (2015). Impact of climate change on agriculture production, food, and nutritional security. Crop Wild Relatives and Climate Change, 1-23.

Yang, A., Cao, S., Yang, Z., Cai, Y., \& Zheng, Y. (2011). $\gamma-$ Aminobutyric acid treatment reduces chilling injury and activates the defence response of peach fruit. Food Chemistry, 129(4), 1619-1622.

Yong, B., Xie, H., Li, Z., Li, Y. P., Zhang, Y., Nie, G., et al. (2017). Exogenous application of GABA improves PEG-induced drought tolerance positively associated with GABA-shunt, polyamines, and proline metabolism in white clover. Frontiers in Physiology, 8, 1107.

Yoshimura, M., Toyoshi, T., Sano, A., Izumi, T., Fujii, T., Konishi, C., et al. (2010). Antihypertensive effect of a $\gamma$-aminobutyric acid rich tomato cultivar 'DG03-9' in spontaneously hypertensive rats. Journal of Agricultural and Food Chemistry, 58, 615-619.

Zhao, Y., Song, X., Zhong, D. B., Yu, L., \& Yu, X. (2020). $\gamma$ Aminobutyric acid (GABA) regulates lipid production and cadmium uptake by Monoraphidium sp. QLY-1 under cadmium stress. Bioresource Technology, 297, 122500.

Zhu, X., Liao, J., Xia, X., Xiong, F., Li, Y., Shen, J., et al. (2019). Physiological and iTRAQ-based proteomic analyses reveal the function of exogenous $\gamma$-aminobutyric acid (GABA) in improving tea plant (Camellia sinensis L.) tolerance at cold temperature. BMC Plant Biology, 19, 43.

Publisher's Note Springer Nature remains neutral with regard to jurisdictional claims in published maps and institutional affiliations. 